18,12

\title{
Модельные оценки свойств флюорографена
}

\author{
() С.Ю. Давыдов
}

Физико-технический институт им. А.Ф. Иофрфе РАН,

Санкт-Петербург, Россия

E-mail: Sergei_Davydov@mail.ru

Поступила в Редакцию 30 августа 2020 г.

В окончательной редакции 30 августа 2020 г.

Принята к публикации 2 сентября 2020 г.

Методом функций Грина в приближении сильной связи получены аналитические выражения для дисперсии электронных зон. Предложена параболическая аппроксимация спектра, в рамках которой определены значения эффективной массы носителей и квантовой емкости. С использованием моделей Костера-Слэтера и Халдейна-Андерсона рассмотрена задача о локальных состояниях моно- и бивакансии. Приведены оценки характерных фононных частот и упругих постоянных. Полученные результаты сопоставлены с данными расчетов других авторов.

Ключевые слова: эффективнае масса, квантовая емкость, вакансии, фононные частоты, упругие постоянные.

DOI: 10.21883/FTT.2021.01.50416.179

\section{1. Введение}

Флюорографен (фтористый графен), представляет собой графен, каждая $p_{z}$-орбиталь которого насыщена атомами фтора, расположенными в чередующемся порядке по обеим сторонам углеродной двумерной (2D) решетки (плоскость $(x, y))$, был, как и графан, сперва теоретически предсказан $[1,2]$ и вскоре синтезирован [3-5]. Свойства флюорографена - полупроводника с широкой запрещенной зоной $\left(E_{g} \sim 3 \mathrm{eV}[3]\right)$ описаны в обзорных работах [6,7]. Что касается теории, то все работы по этой тематике являются численными расчетами, использующими различные варианты DFT (density functional theory). Имея в виду возможное использование флюорографена в приборных структурах, хотелось бы иметь простую аналитическую теорию, описывающую его свойства. В работе [8] нами были предложены достаточно простые схемы расчета электронных и фононных характеристик графана, которую мы используем здесь и для флюорографена.

\section{2. Электронный спектр}

Рассмотрим димер $\mathrm{C}-\mathrm{F}$, в котором взаимодействие между атомами углерода и фтора описывается матричным элементом $t_{\perp}$. Соответствующие функции Грина такого димера имеют вид

$$
G_{\mathrm{C}(\mathrm{F})}(\omega)=\frac{g_{\mathrm{C}(\mathrm{F})}(\omega)}{1-g_{\mathrm{C}}(\omega) g_{\mathrm{F}}(\omega) t_{\perp}^{2}},
$$

где $\omega-$ энергетическая переменная, $g_{\mathrm{C}(\mathrm{F})}(\omega)=$ $=\left(\omega-\varepsilon_{c(f)}+i 0^{+}\right)^{-1}-$ функция Грина изолированного атома углерода (фтора) с энергией $p$-орбитали $\varepsilon_{c(f)}$. Теперь построим из этих димеров гексагональную
2D-решетку, включив взаимодействие $t$ между ближайшими углеродными атомами димеров. Тогда получим функции Грина флюорографена в виде

$$
\tilde{G}_{\mathrm{C}}(\omega, \mathbf{k})=\frac{G_{\mathrm{C}}(\omega)}{1-G_{\mathrm{C}}^{2}(\omega) t^{2} f^{2}(\mathbf{k})},
$$

где $f(\mathbf{k})=\sqrt{3+2 \cos \left(k_{x} a \sqrt{3}\right)+4 \cos \left(k_{x} a \sqrt{3}\right) \cos \left(3 k_{y} a / 2\right)}$ (ось $x$ направлена вдоль зигзагообразной кромки), $a=1.58 \AA$ [7] — расстояние между ближайшими атомами углерода, $\mathbf{k}=\left(k_{x}, k_{y}\right)-$ волновой вектор для движения электрона в плоскости $(x, y)$. Легко показать [8], что электронный спектр флюорографана есть

$$
\begin{gathered}
E_{1}(\mathbf{k})=\frac{1}{2}\left(\sqrt{[|\varepsilon|+t f(\mathbf{k})]^{2}+4 t_{\perp}^{2}}+t f(\mathbf{k})\right) \\
=-E_{4}(\mathbf{k}), \\
E_{2}(\mathbf{k})=E_{C}(\mathbf{k})=\frac{1}{2}\left(\sqrt{[|\varepsilon|-t f(\mathbf{k})]^{2}+4 t_{\perp}^{2}}-t f(\mathbf{k})\right) \\
=-E_{3}(\mathbf{k})=-E_{V}(\mathbf{k}),
\end{gathered}
$$

где мы положили $\varepsilon_{c}=-\varepsilon_{f}=\varepsilon$; индексы $1-4$ нумеруют зоны, расположенные по убыванию их энергии, а индексы $C$ и $V$ отвечают соответственно зоне проводимости и валентной зоне. Характерные энергетические щели спектра можно представить в следующем виде ( $Г, \mathrm{~K}, \mathrm{M}$ - точки зоны Бриллюэна): $\Delta_{\mathrm{K}}=E_{C}(\mathrm{~K})-E_{V}(\mathrm{~K})=r_{0}, r_{0}=\sqrt{\varepsilon^{2}+4 t_{\perp}^{2}}, \Delta_{\mathrm{M}}=E_{C}(\mathrm{M})$ $-E_{V}(\mathrm{M})=r_{2-}-2 t, r_{2 \pm}=\sqrt{(\varepsilon \pm 2 t)^{2}+4 t_{\perp}^{2}}, \Delta_{\Gamma}=E_{g}=$ $=E_{C}(\Gamma)-E_{V}(\Gamma)=r_{3-}-3 t, r_{3 \pm}=\sqrt{(\varepsilon \pm 3 t)^{2}+4 t_{\perp}^{2}}[8]$. Согласно результатам расчетов [2,9-11], имеем: $E_{g}=$ $=2.75-3.2 \mathrm{eV}, \Delta_{\mathrm{K}} \sim 10-11 \mathrm{eV}, \Delta_{\mathrm{M}} \sim 7-8 \mathrm{eV}$. Полагая 
$E_{g}=3 \mathrm{eV}, \Delta_{\mathrm{K}}=10 \mathrm{eV}, t=2.8 \mathrm{eV}$, получим $\varepsilon=2.40 \mathrm{eV}$, $t_{\perp}=4.85 \mathrm{eV}$. Далее, как и в [8], аппроксимируем зоны (3) и (4) следующими выражениями:

$$
\begin{aligned}
& E_{C}(\mathbf{k})=-E_{V}(\mathbf{k})=\frac{E_{g}}{2}+\frac{\hbar^{2} k^{2}}{2 m_{e}}, \\
& E_{1}(\mathbf{k})=-E_{4}(\mathbf{k})=\frac{E_{g}}{2}-\frac{\hbar^{2} k^{2}}{2 m_{1}},
\end{aligned}
$$

где электронные эффективные массы равны

$$
\begin{aligned}
& m_{e}=\frac{4 \hbar^{2}}{3 a^{2} t}\left(1-\frac{3 t-\varepsilon}{r_{3}}\right)^{-1}, \\
& m_{1}=\frac{4 \hbar^{2}}{3 a^{2} t}\left(1+\frac{3 t+\varepsilon}{r_{3+}}\right)^{-1} .
\end{aligned}
$$

Полагая длину связи С-C равной $a=1.58 \AA$ [7], получим $\quad m_{e}^{*}=m_{e} / m_{0} \approx 3.1, \quad m_{1}^{*}=m_{1} / m_{0} \approx 0.8, \quad$ где $m_{0}$ - масса свободного электрона. В случае графана $m_{e}^{*}=m_{e} / m_{0} \approx 1.9, m_{1}^{*}=m_{1} / m_{0} \approx 1.2$ [8]. Отметим, что доказательства приемлемости параболической аппроксимации спектра, приведенные для графана в [8] (см. рис. 2), справедливы и в настоящем случае.

\section{3. Квантовая емкость}

Обратимся к оценке квантовой емкости флюорографена $C_{Q}$, выражение для которой удобно представить в виде [12]:

$$
\begin{gathered}
C_{Q}\left(V^{*}\right)=\left(e^{2} / 4 T S\right) \int_{0}^{\infty} \rho(\omega) A\left(\omega, V^{*}\right) d \omega, \\
A\left(\omega, V^{*}\right)=\operatorname{sch}^{2}\left[\left(\omega-V^{*}\right) / 2 T\right]+\operatorname{sch}^{2}\left[\left(\omega+V^{*}\right) / 2 T\right],
\end{gathered}
$$

где $V^{*}=e V_{e l}$ - сдвиг электронных состояний в электростатическом поле, $V_{e l}$ - внешний электростатический потенциал, $e-$ величина заряда электрона, $T-$ температура в энергетических единицах, $S$ - площадь, приходящаяся на один атом углерода. В (7) предполагается, что при $V^{*}=0$ химический потенциал совпадает с центром запрещенной зоны флюорографена.

В двумерных системах спектрам отвечают плотности состояний $\rho(\omega)=$ const [13]. Как и в [8], положим $\rho_{C}(\omega)=\rho_{V}(\omega)=2 / W_{C}$ при $E_{C}(\Gamma) \leq|\omega| \leq E_{C}(\mathrm{~K})$, где $W_{C}=W_{V}=\left(3 t+r_{0}-r_{3}\right) / 2=3.50 \mathrm{eV}-$ ширина зон проводимости и валентной (модель типа ,пьедестал“); $\rho_{1}(\omega)=\rho_{4}(\omega)=2 / W_{1}$ при $E_{1}(\mathrm{~K})=$ $=E_{C}(\mathrm{~K}) \leq|\omega| \leq E_{1}(\Gamma)$, где $W_{1}=W_{4}=\left(3 t+r_{3+}-r_{0}\right) / 2$ $=6.5 \mathrm{eV}$. В приближении средней ширины зоны (см. подробнее [8]) при комнатной температуре получим окончательно

$$
C_{Q}\left(V^{*}\right) \approx \frac{4 e^{2} \operatorname{ch}\left(V^{*} / T\right)}{S \bar{W}} \exp \left(-E_{g} / 2 T\right) .
$$

Квантовая емкость $C_{Q}^{0} \equiv C_{Q}(0) \approx(16 / 3 \sqrt{3})\left(e^{2} / \bar{W} a^{2}\right)$ $\times \exp \left(-E_{g} / 2 T\right)$, так что для комнатной температуры получаем $C_{Q}^{0}=0$ (для графена $C_{Q}^{0} \approx 16 \mu \mathrm{F} / \mathrm{cm}^{2}$ [15]); $C_{Q}\left(E_{g} / 2\right) \approx(8 / 3 \sqrt{3})\left(e^{2} / \bar{W} a^{2}\right)=0.2 \mathrm{mF} / \mathrm{cm}^{2} \quad$ (как и у графана $[8])$.

\section{4. Локальные состояния вакансий}

Перейдем теперь к оценкам электронных характеристик вакансий в флюорографене, воспользовавшись для этого моделями Костера-Слэтера и Халдейна-Андерсона $[8,15]$. В первой из этих моделей предполагается, что возмущение, вносимое дефектом, сосредоточено в одном узле решетки и равно $V$, а локальные состояния, лежащие в запрещенной зоне $\left(\left|w_{l}\right|<E_{g} / 2\right)$, определяются (после ряда упрощений) уравнением

$$
\frac{2 V}{\bar{W}} \ln \left(\frac{E_{g} / 2-\omega}{E_{g} / 2+\omega}\right) \approx 1 .
$$

Плотности состояний этих уровней $\rho_{l}(\omega)$ и числа их заполнения $n_{l}$ при $T=0$ равны $\rho_{l}(\omega)=v_{l} \delta\left(\omega-\omega_{l}\right)$ и $n_{l}=v_{l} \Theta\left(E_{F}-\omega_{l}\right)$, где $\delta(\ldots)$ - функция Дирака, $\Theta(\ldots)-$ функция Хэвисайда, $E_{F}-$ уровень Ферми, а факторы заполнения $v_{l}$ есть

$$
v_{l} \approx \frac{\bar{W}\left[\left(E_{g} / 2\right)^{2}-\omega^{2}\right]}{2 E_{g} V^{2}} .
$$

Свойства модели Костера-Слэтера подробно обсуждаются в $[8,15]$. Здесь отметим только, что в этой модели предел $|V| \rightarrow \infty$, при котором $\omega \rightarrow 0$ и $v_{l} \rightarrow 0$, соответствует наличию вакансии в матрице. При этом углеродная вакансия означает удаление димера С-H в случае графана и димера $\mathrm{C}-\mathrm{F}$ в случае флюорографена.

По данным [16], энергия одиночной углеродной вакансии в флюорографене $\omega_{l}^{c}=0.78 \mathrm{eV}$. Тогда из (9) получим $V^{c}=-2.2 \mathrm{eV}$, а из $(10)$ находим $v_{l} \approx 0.3$. То же значение $v_{l}$ получено в [8] для графана. В случае вакансии фтора $\omega_{l}^{f}=-0.17[16]$, так что аналогичным образом получаем $V^{f}=11 \mathrm{eV}$ и $v_{l} \approx 0$. Здесь следует, однако, отметить, что в работе [16] зонная структура флюорографена рассматривалась в многочастичном варианте DFT, в результате чего рассчитанное значение $E_{g}$ в два раза превысило экспериментальную величину [3] (ситуация, типичная в такого рода расчетах — см., например, $[17,18])$. Так как в настоящей работе ширина запрещенной зоны принята равной $3 \mathrm{eV}$, полученные значения параметров $V$ и $v_{l}$ нужно рассматривать лишь как достаточно грубые оценки.

Модель Халдейна-Андерсона, по сравнению с моделью Костера-Слэтера, во-первых, содержит дополнительный параметр $\varepsilon_{d}-$ уровень дефекта. Во-вторых, потенциал $V_{d}$ взаимодействия дефекта $\mathrm{c}$ матрицей принципиально отличается от возмущения $V$ модели Костера-Слэтера: первый отвечает туннелированию 
электрона между уровнем дефекта $\varepsilon_{d}$ и зонными состояниями матрицы, тогда как второй описывает возмущение, вызванное заменой атома матрицы на чужеродный атом [15]. В упрощенном варианте (см. подробнее [8]) энергия дефектного состояния в запрещенной зоне $\omega_{l}$ определяется корнем уравнения

$$
\omega-\varepsilon_{d}-\frac{2 V_{d}^{2}}{\bar{W}} \ln \left(\frac{E_{g} / 2-\omega}{E_{g} / 2+\omega}\right) \approx 0,
$$

а фактор заполнения есть

$$
v_{l}=\left(1+\frac{2 E_{g} V_{g}^{2}}{\bar{W}\left[\left(E_{g} / 2\right)^{2}-\omega^{2}\right]}\right)^{-1} .
$$

Положив для простоты $V_{d}=t$ [8] и вновь воспользовавшись значением энергетического уровня вакансии фтора $\omega_{l}^{f}=-0.17 \mathrm{eV}[16]$, получим из (12) фактор заполнения $v_{l}^{h} \approx 0.19$, а из $(11)$ найдем $\varepsilon_{d} \approx-0.88 \mathrm{eV}$.

Рассмотрим теперь ситуацию, когда удалены два атома фтора, связанные изначально с соседними атомами углерода, или бивакансию. В рамках модели Халдейна-Андерсона такую ситуацию можно представить как взаимодействующий с флюорогрефаном дополнительный (C-C)-димер. Функция Грина такого „примесного димера“ равна $G_{\operatorname{dim}}(\omega)=g_{d}(\omega)\left[1-g_{d}^{2}(\omega)\right]^{-1}$, где $g_{d}^{-1}(\omega)=\omega-\varepsilon_{d}-\Lambda(\omega)+i \Gamma(\omega)-$ функция Грина моновакансии $[8,19]$. Таким образом, энергетические уровни бивакансии определяются корнями уравнений

$$
\omega-\varepsilon_{d}-\frac{2 V_{d}^{2}}{\bar{W}} \ln \left(\frac{E_{g} / 2-\omega}{E_{g} / 2+\omega}\right) \pm t \approx 0 .
$$

В результате получаем: $\omega_{l-}^{f} \approx-0.67 \mathrm{eV}, v_{l-}^{f}=0.16$; $\omega_{l+}^{f} \approx 0.36 \mathrm{eV}, v_{l+}^{f}=0.18$. Отметим, что в [20] pacсматривалась принципиальная возможность использования аналогичной двухуровневой системы, созданной водородной бивакансией в графане, для квантовых вычислений.

\section{5. Фононные частоты и упругие постоянные}

Обратимся теперь к оценкам упругих и фононных характеристик флюорографена, для чего воспользуемся моделью связывающих орбиталей Харрисона [21,22]. Энергию $E_{b}$ связи $\mathrm{C}-\mathrm{H}$ и соответствующую силовую константу центрального взаимодействия $k_{0 \perp}$ можно представить в виде

$$
\begin{gathered}
E_{b}=2 V_{2} \alpha_{c}^{-1}\left(1-2 \alpha_{c} / 3\right), \\
k_{0 \perp}=4 \alpha_{c} V_{2}\left(1-2 \alpha_{p}^{2}\right) / a_{\perp}^{2},
\end{gathered}
$$

где $V_{2}-$ ковалентная энергия, $\alpha_{c}=V_{2} / \sqrt{V_{2}^{2}+V_{3}^{2}}$ и $\alpha_{p}=\sqrt{1-\alpha_{c}^{2}}-$ ковалентность и полярность связи $\mathrm{C}-\mathrm{F}$ (длина которой равна $\left.a_{\perp}=1.4 \AA[6]\right), V_{3}-$ по- лярная энергия (см. подробнее [21-23]). Полагая, что имеет место $\sigma$-связь $p$-орбиталей атомов $\mathrm{C}$ и $\mathrm{F}$, имеем $V_{2}=V_{p p \sigma}=\eta_{p p \sigma}\left(\hbar^{2} / m_{0} a_{\perp}^{2}\right)$, где $\eta_{p p \sigma}=2.22$ [22], так что $V_{2}=8.63 \mathrm{eV}$. Полярная энергия $V_{3}=\left(\varepsilon_{c p}-\varepsilon_{f p}\right) / 2$, где $\varepsilon_{c p}=-11.07 \mathrm{eV}$ - энергия $p$-состояния углерода и $\varepsilon_{f p}=-19.87 \mathrm{eV}$ - энергия $p$-состояния фтора [24], равна $4.40 \mathrm{eV}$, так что $\alpha_{c}=0.89$ и $\alpha_{p}=0.45$. Из (14) получим $E_{b}=7.89 \mathrm{eV}$ (в [3] приводится значение $5.3 \mathrm{eV}$ ) и $k_{0 \perp}=9.3 \mathrm{eV} / \AA$. Характерная частота нормальных колебаний атома фтора с массой $M_{\mathrm{F}}$ относительно неподвижного углеродного листа (см. подробнее [8]) равна $\Omega_{\perp}=\sqrt{k_{0 \perp} / M_{\mathrm{F}}}=0.045 \mathrm{eV}=364 \mathrm{~cm}^{-1}$. Такая частота перекрываются с зоной акустических фононов флюорографена (от 0 до $\sim 1100 \mathrm{~cm}^{-1}$ ) [25]. В случае графана частота колебания атома водорода $\Omega_{\perp}=\sqrt{k_{0 \perp} / m_{p}}$ ( $m_{p}$ - масса протона) равна $0.344 \mathrm{eV}$, или $2774 \mathrm{~cm}^{-1}$ [8], что совпадает с полученным в [25] значением. Столь существенное различие частот объясняется большой разницей масс атомов $\mathrm{F}$ и $\mathrm{H}$.

Если считать, что $\sigma$-связь атомов $\mathrm{C}$ и $\mathrm{F}$ осуществляется соответственно $s p^{3}-$ и р-орбиталями, то $V_{2}=V_{p / s p 3}=\eta_{p / s p 3}\left(\hbar^{2} / m_{0} a_{\perp}^{2}\right)$, где $\eta_{p / p s 3}=2.63[26]$, получим $V_{2}=10.22 \mathrm{eV}, \alpha_{c}=0.95$ и $\alpha_{p}=0.31$. Энергия $s p^{3}$-орбитали атома углерода $\varepsilon_{c s p 3}=\left(\varepsilon_{c s}+3 \varepsilon_{c p}\right) / 4$ $=-13.12 \mathrm{eV}$ (таблицы [24]), так что $V_{3}=3.36 \mathrm{eV}$. Получаем: $E_{b}=7.89 \mathrm{eV}, k_{0 \perp}=16.0 \mathrm{eV} / \AA, \Omega_{\perp}=0.059 \mathrm{eV}$ $=475 \mathrm{~cm}^{-1}$.

Оценим также характерную энергию электронфононной связи $\lambda=w^{2} / k_{0 \perp}$, где деформационный фактор $w=-\partial V_{2} / \partial a_{\perp}$ [27]. Так как $-\partial V_{2} / \partial a_{\perp}=2 V_{2} / a_{\perp}$ и $k_{0 \perp} \approx 4 V_{2} / a_{\perp}^{2}$, получим $\lambda \approx V_{2}$. Эта величина гораздо выше, чем значения $\lambda$ для адсорбированных на графене двух-, трех- и четырехатомных молекул газа, адсорбированных на графене [27]. Так как $\lambda \propto a_{\perp}^{-2}$, такой результат объясняется малой длиной связи $\mathrm{C}-\mathrm{F}$. Та же ситуация имеет место для графана [8].

Перейдем к колебаниям в плоскости углеродного листа флюорографена. Оценки показывают, что сделанное в [8] предположение о жесткой связи атомов димера $\mathrm{C}-\mathrm{H}$ в случае димера $\mathrm{C}-\mathrm{F}$ не работает. Воспользуемся поэтому моделью двух связанных осцилляторов, предложенной нами для описания положения G-пика раманспектра в эпитаксиальном графене [28].

Рассмотрим длинноволновые (соответствующие точке Г) оптические колебаний атомов углерода, образующих 2D-гексагональную решетку. Такие колебания отвечают G-пику раман-спектра. Для свободного графена их частота $\bar{\Omega}=1580 \mathrm{~cm}^{-1}$ [29]. Учтем, что в флюорографене длина $\mathrm{C}-\mathrm{C}$-связи $a=1.58 \AA$, тогда как в графене она равна $\bar{a}=1.42 \AA \AA$. Тогда $k_{0} / \bar{k}_{0}=(\bar{a} / a)^{4} \approx 0.65$, если предполагать, что $\sigma$-связи в листах флюорографена и графена образованы орбиталями с одним и тем же типом гибридизации. Если же считать, что для графена имеет место $s p^{2}$-гибридизация, а для флюорогруфана $s p^{3}$-гибридизация, то $k_{0} / \bar{k}_{0}=\left(\eta_{s p 3} / \eta_{s p 2}\right)(\bar{a} / a)^{4} \approx 0.64$, где $\eta_{s p 2}=3.26$ и $\eta_{s p 3}=3.22$ [22]). Тогда (без учета 
Результаты оценок безразмерных эффективных масс $m_{C}^{*}$ и $m_{1}^{*}$, квантовых емкостей $C_{\Omega}^{0}$ в ед. $\mu \mathrm{F} / \mathrm{cm}^{2}$, фононных частот $\Omega_{\perp}$ и $\Omega$ в $\mathrm{cm}^{-1}$, силовых констант $k_{0 \perp}, \alpha, \beta$ в ед. $\mathrm{N} / \mathrm{m}$ и отношения $k_{0} / \bar{k}_{0}$, где $\bar{k}_{0}-$ силовая константа центрального взаимодействия графена, для флюорографена (FGr), графана (HGr) [8] и графена (Gr) [14,23].

\begin{tabular}{c|c|c|c|c|c|c|c|c}
\hline Параметры & $m_{C}^{*}$ & $m_{1}^{*}$ & $C_{Q}^{0}$ & $\Omega_{\perp}$ & $\Omega$ & $k_{0 \perp}$ & $k_{0} / \bar{k}_{0}$ & $\alpha, \beta$ \\
\hline FGr & 3.1 & 0.8 & 0.7 & 364 & 1280 & 9.3 & 0.65 & 41,53 \\
& & & & 475 & 1268 & 16.0 & & \\
\hline HGr & 1.9 & 1.2 & 0.7 & 2774 & 1325 & 28.5 & 0.76 & 43,59 \\
& & & & 3230 & 1317 & 38.6 & 0.75 & \\
\hline Gr & - & - & 1.6 & - & 1580 & - & 1 & 77,69
\end{tabular}

Примечание. Для частот $\Omega \perp, \Omega$, силовой константы $k_{0 \perp}$ и отношения $k_{0} / \bar{k}_{0}$ приводятся два значения, зависящие от гибридизации орбиталей, образующих $\sigma$-связи $\mathrm{C}-\mathrm{F}$ и $\mathrm{C}-\mathrm{H}$ (см. текст). В качестве $\Omega$ для графена приведено экспериментальное значение частоты G-пика раман-спектра.

атомов фтора), для частоты колебаний $\Omega$ связи $\mathrm{C}-\mathrm{C}$ получим соответственно $\Omega / \bar{\Omega}=(\bar{a} / a)^{2} \approx 0.81$ и $\Omega / \bar{\Omega}=(\bar{a} / a)^{2} \sqrt{\eta_{s p 3} / \eta_{s p 2}} \approx 0.80$, т. е. $\Omega \approx 1280 \mathrm{~cm}^{-1}$ и $\Omega \approx 1268 \mathrm{~cm}^{-1}$. Для графана в [8] эти частоты равны $1325 \mathrm{~cm}^{-1}$ при $s p^{2}$-гибридизации и $1317 \mathrm{~cm}^{-1}$ при $s p^{3}$-гибридизации. Как для графана, так и для флюорографена полученные значения частот вполне удовлетворительно согласуются с результатами работы [25].

Учтем теперь связь между колебаниями $\mathrm{C}-\mathrm{F}$ и $\mathrm{C}-\mathrm{C}$, которую будем описывать силовой константой $\kappa$. Воспользовавшись результатами [28] и полагая для простоты $\kappa \ll k_{0}, k_{0 \perp}$, получим перенормированные за счет взаимной связи значения частот $\widetilde{Q}_{\perp}$ и $\widetilde{\Omega}$ :

$$
\widetilde{\Omega}_{\perp} \approx \Omega_{\perp}\left(1+\kappa / 2 k_{0 \perp}\right), \quad \widetilde{\Omega} \approx \Omega\left(1+\kappa / 2 k_{0}\right) .
$$

Таким образом, учет связи колебаний приближает частоты $\widetilde{\Omega}$ флюорографена к частотам $\Omega$ графана [8].

Упругие постоянные $c_{11}$ и $c_{12}$, полученные в рамках модели Китинга для графена $[8,23]$ и описывающие реакцию гексагональной двумерной решетки на деформацию в плоскости листа, имеют вид

$$
\begin{aligned}
& c_{11}=\frac{1}{\sqrt{3}}\left(4 \alpha+\beta+18 \frac{\alpha \beta}{4 \alpha+\beta}\right), \\
& c_{12}=\frac{1}{\sqrt{3}}\left(4 \alpha+\beta-18 \frac{\alpha \beta}{4 \alpha+\beta}\right),
\end{aligned}
$$

где $\alpha$ и $\beta$ - силовые константы центрального и нецентрального взаимодействий. Значения силовых констант определим из рассчитанных в [9] модуля Юнга $E=\left(c_{11}^{2}-c_{12}^{2}\right) / c_{11}=226 \mathrm{~N} / \mathrm{m}$, коэффициента Пуассона $\sigma=c_{12} / c_{11}=0.10$ и упругих постоянных $c_{11}=228 \mathrm{~N} / \mathrm{m}$ и $c_{12}=23 \mathrm{~N} / \mathrm{m}$. (Отметим, однако, что в единственной, насколько известно автору, экспериментальной работе [3] приведено значение $E=100 \mathrm{~N} / \mathrm{m})$. Из (16) получаем $\alpha=41 \mathrm{~N} / \mathrm{m}$ и $\beta=53 \mathrm{~N} / \mathrm{m}$. Как и в случае графана [8], отношение $\beta / \alpha>1$; для графена $\beta / \alpha=0.90$ [23].
В случае классических полупроводников типа $\mathrm{A}_{\mathrm{N}} \mathrm{B}_{8-\mathrm{N}}$ отношение $\beta / \alpha<1$ и уменьшается с ростом ионности связи [30].

Следует отметить, что центральная силовая константа $k_{0 \perp}$ описывает реакцию графана на сжатие или растяжение связи в направлении, перпендикулярном листу, и поэтому является одномерным аналогом объемного модуля сжатия. Полученные нами здесь значения $k_{0 \perp}=9.3 \mathrm{~N} / \mathrm{m} \quad$ (связь $p-p$ ) и $16 \mathrm{~N} / \mathrm{m}$ (связь $\left.s p^{3}-p\right)$ для флюорографена значительно ниже значений $k_{0 \perp}=28.5 \mathrm{~N} / \mathrm{m}($ связь $p-s)$ и $38.6 \mathrm{~N} / \mathrm{m}\left(\right.$ связь $\left.s p^{3}-s\right)$ для графана [8].

\section{6. Заключение}

Основные результаты работы сведены в таблицу. Из таблицы следует, что главное различие между флюорографеном и графаном - значения частот $\Omega_{\perp}$, причем это различие продиктовано разницей как силовых констант $k_{0 \perp}$, так и (в большей степени) масс атомов фтора и водорода. Второе по величине различие- значения эффективных масс. В остальном, следует отметить близость характеристик флюорографена и графана.

Отметим, что интерес к графану и флюорографену является проявлением общей тенденции - поиска (в большей степени, теоретического) новых 2D материалов, которые могут представить интерес для наноэлектроники [31-33]. Поэтому, вообще говоря, можно было бы обобщить результаты настоящей работы и работы [8] на флюорографено- и графаноподобные структуры, в которых вместо атомов углерода в узлах гексагональной 2D решетки находятся атомы А и В. Отметим, что для реализации этой программы в [8] уже получено уравнение для нахождения соответствующих электронных зон, а в [23] приведены формулы, необходимые для расчетов силовых констант и, как следствие, характерных фононных частот. Нам, однако, подобная работа представляется преждевременной из-за достаточно ограниченной экспериментальной информации по исходным соединениям - графану и флюорографену.

\section{Конфликт интересов}

Автор заявляет об отсутствии конфликта интересов.

\section{Список литературы}

[1] J.O. Sofo, A.S. Chaudhari, G.D. Barber. Phys. Rev. B 75, 153401 (2007).

[2] O. Leenaerts, H. Peelaers, A.D. Hernández-Nieves, B. Partoens, F.M. Peeters. Phys. Rev. B 82, 195436 (2010).

[3] R.R. Nair, W. Ren, R. Jalil, I. Riaz, V.G. Kravets, L. Britnell, P. Blake, F. Schedin, A.S. Mayorov, S. Yuan, M.I. Katsnelson, H.-M. Cheng, W. Strupinski, L.G. Bulusheva, A.V. Okotrub, I.V. Grigorieva, A.N. Grigorenko, K.S. Novoselov, A.K. Geim. Small 6, 2877 (2010). 
[4] R. Zboril, F. Karlicky, A.B. Bourlinos, T.A. Steriotis, A.K. Stubos, V. Georgakilas, K. Safarova, D. Jancik, C. Trapalis, M. Otyepka. Small 6, 2885 (2010).

[5] K.-J. Jeon, Z. Lee, E. Pollak, L. Moreschini, A. Bostwick, C.-M. Park, R. Mendelsberg, V. Radmilovic, R. Kostecki, T.J. Richardson, E. Rotenberg. ACS Nano 5, 1042 (2011).

[6] W. Feng, P. Long, Y. Feng, Y. Li. Adv. Sci. 3, 1500413 (2016). DOI: 10.1002/advs.201500413.

[7] D.D. Chronopoulos, A. Bakandritsos, M. Pykal, R. Zboril, M. Otyepka. Appl. Mater. Today 9, 60 (2017).

[8] С.Ю. Давыдов. ФТТ 62, 2151 (2020).

[9] E. Muñoz, A.K. Singh, M.A. Ribas, E.S. Penev, B.I. Yakobson. Diamond Rel. Mater. 19, 368 (2010).

[10] D.K. Samarakoon, Z. Chen, C. Nicolas, X.-Q. Wang. Small 7, 965 (2011).

[11] W. Wei, T. Jacob. Phys. Rev. B 87, 115431 (2013).

[12] D.L. John, L.C. Castro, D.L. Pulfrey. J. Appl. Phys. 96, 5180 (2004).

[13] T. Ando, A.B. Fowler, F. Stern. Rev. Mod. Phys. 54, 437 (1982).

[14] С.Ю. Давыдов, А.А. Лебедев, П.В. Булат, А.В. Зубов. Письма в ЖТФ 46, 15, 7 (2020).

[15] С.Ю. Давыдов, А.А. Лебедев, О.В. Посредник. Элементарное введение в теорию наносистем. Изд-во „Лань“, СПб (2014).

[16] S. Yuan, M. Rösner, A. Schulz, T.O. Wehling, M.I. Katsnelson. Phys. Rev. Lett. 114, 047403 (2015).

[17] W. Wei, T. Jacob. Phys. Rev. B 87, 115431 (2013).

[18] F. Karlicky, M. Otyepka. Ann. Phys. (Berlin) 526, 408 (2014).

[19] С.Ю. Давыдов. ФТТ 54, 2193 (2012).

[20] L. Hao, H.-Yan Lu, C.S. Ting. Phys. Rev. Mater. 3, 024003 (2019); arXiv: 1812.0777v1.

[21] У. Харрисон. Электронная структура и свойства твердых тел. Мир, М.(1983).

[22] W.A. Harrison. Phys. Rev. B 27, 3592 (1983).

[23] С.Ю. Давыдов, О.В. Посредник. ФТТ 57, 819 (2015).

[24] W.A. Harrison. Phys. Rev. B 31, 2121 (1985).

[25] H. Peelaers, A.D. Hernández-Nieves, O. Leenaerts, B. Partoens, F.M. Peeters. Appl. Phys. Lett. 98, 051914 (2011).

[26] С.Ю. Давыдов, Г.И. Сабирова. Письма в ЖТФ 37, 11, 51 (2011).

[27] С.Ю. Давыдов. Письма в ЖТФ 44, 3, 40 (2018).

[28] С.Ю. Давыдов. ФТТ 59, 610 (2017).

[29] Z.H. Ni, W. Chen, X.F. Fan, J.L. Kuo, T. Yu, A.T.S. Wee, Z.X. Shen. Phys. Rev. B 77, 115416 (2008),

[30] J.C. Phillips. Rev. Mod. Phys. 42, 317 (1970).

[31] A.K. Geim, I.V. Grigorieva. Nature 499, 419 (2013).

[32] C.-J. Tong, H. Zhang, Y.-N. Zhang, H. Liu, L.-M. Liu. J. Mater. Chem. A 2, 17971 (2014).

[33] И.В. Антонова. ФТП 50, 67 (2016).

Редактор К.В. Емиев 